\title{
A poésie numérique un objet d'essai triadique
}

Wilton Azevedo

\section{Université Presbiterian Mackenzie}

wiazevedeterra.com.br

\begin{abstract}
Résumé : Dans cette ambiance nous observons, dans les dernières années, qu'il n'y a pas de collision de codes. Nous voyons leur mouvement d'expansion - l'écriture en expansion. La sémiotique de C. S. Peirce (1977), en l'acceptant comme une triade, qui part vers un système rhizomatique, pas tout à fait dual et moins encore diachronique, ce qui dans la pensée de Peirce finit par déconsidérer le sens manichéiste entre : l'erreur et la justesse, l'étrangeté et la répétition, l'abstrait et le figuratif. Si nous regardons donc l'action de n'importe quel signe, il sera toujours immensurable et il ne peut y avoir, dans son processus d'expansion, cette division où un signe engendre un autre signe.
\end{abstract}

Quelques indices nos signalent, pendant ces années-là, que les systèmes de langage programmatique formatent notre planète de façon à nous tendre des pièges, car l'implantation de l'internet, les portables, les TV plasma interactives ont fini par apporter un nouveau sens au verbe «lire ». Cet état éphémère nous fait perdre la dimension de l'écriture hypostatique et comme il ne s'agit pas d'un texte de rhétorique linéaire, les changements culturels se font dans la façon que l'humanité passera à acquérir les connaissances.

La poésie numérique et surtout le poème numérique servent d'expérimentation, en entrainant de nouveaux récits et discours dans l'hypermédia - que j'appelle Interpoesial - pour que, dans le déroulement de la pensée du parcours des signes plongés dans cette ambiance de l'écriture numérique mettent en question le sens de la compréhension et de l'éloquence.

1 Interpoesia CD-ROM, Wilton Azevedo \& Phildelpho Meneses, FAPESP, EDTORA MAKENZIE, à São Paulo, en 2000. 
Dans cette ambiance nous observons, dans les dernières années, qu'il n'y a pas de collision de codes. Nous voyons leur mouvement d'expansion - l'écriture en expansion - qui n'appartient plus à de codes isolés dans leur espace temporel, mais à un temps/espace de signes, qui travaille avec l'articulation et le registre de signes multi-dimensionnés en espace/temps, lequel n'est plus bidimensionnel, paradigmatique, formé par des processus syntagmatiques.

C'est pourquoi on doit reprendre la sémiotique de C. S. Peirce $(1977)^{2}$, en l'acceptant comme une triade, qui part vers un système rhizomique, pas tout à fait dual et moins encore diachronique, ce qui dans la pensée de Peirce finit par déconsidérer le sens manichéiste entre : l'erreur et la justesse, l'étrangeté et la mesmice l'abstrait et le figuratif. Si nous regardons donc l'action de n'importe quel signe, il sera toujours immensurable et il ne peut avoir, dans son processus d'expansion, cette division où un signe engendre un autre signe.

Dans la poésie numérique, les codes ne se divisent plus diachroniquement dans le concept de mesmice et d'étrangeté, de fiction et de réalité, comme prévu dans les manifestes du siècle dernier. On a insisté tellement sur ces ruptures que l'art a fini par créer, dans l'invention de la performance, quelque chose qui rendait le langage poétique vulnérable au

2 Pour Charles Sanders Peirce l'abduction est le processus de formation d'une hypothèse déclaratoire. C'est la seule opération logique qui présente une nouvelle idée, car l'induction n'a rien fait outre déterminer une valeur, et la déduction pure et, simplement développe les conséquences nécessaires d'une hypothèse pure. (Peirce, C. S., 1977 page 220). 
registre historique - hic et nunc - et ainsi, toute et n'importe quelle manifestation artistique ne demeure plus au service, ni de la fiction, ni de la réalité : elle serait valable uniquement réalisée dans le présent- real time. De cette façon, la poésie numérique devra être revue, en tant qu'environnement de signes qui s'articulent, ainsi que la parole et la musique, en opérant dans une somation d'environnements : l'ambiance. Dans le sens sémiotique, le «faire signe » de la poésie numérique devient visible d'une manière indicielle par rapport au modèle mathématique adopté.

Pour le développement de la poésie numérique, il faut qui nous nous concentrons sur une révision concernant la poésie et la littérature qui employait déjà la syntaxe non linéaire parataxe - comme une actualisation des préceptes connus par le domaine des lettres, mais avec une nouvelle notion de rythme, de récit et de discours. L'espace délimité par le temps et l'espace littéraire et poétique (Zumthor, 2007), issu de la pratique des rhapsodes du Moyen Âge, est quelque chose qui vient de très loin et s'est accentuée pendant le XVIIe et le XVIII siècles jusqu'à nos jours. La poésie qui acquiert, à partir de cette caractéristique historique, une culture anthropologique, est mise en question face à la poésie programmatique.

La grande dichotomie, qui doit alors être surmontée, commencerait avec : l'oral/l'écrit (Zumthor, 2007. Pag.13), par l'imagé/le canonique et le sonore/le chiffré, où tout espace numérique, vu qu'il opère dans les concepts de la simulation, nous transmet une sensation d'artificialité. Cependant, tout ce qui passe par notre cognition - raison perceptive - ne peut être considéré comme simple abstraction : 
Il me semble, tout au moins, que je peux dire : ce qui se perd tout de même avec les médias, et qui demeurera nécessairement ainsi, c'est la corporéité, le poids, la chaleur, le volume réel du corps, dont la voix n'est qu'une expansion (Zumthor, 2007 page 16).

Je suis en partie d'accord avec Zumthor, car à chaque jour le monde numérique se corporifie aussi dans sa manière d'accès et d'expression, et ce n'est pas seulement la voix - le son qui gagne son expansion de signe, mais n'importe quel code, qui avait déjà, avant de devenir logiciel, des caractéristiques virtuelles, comme c'est le cas de la parole. Si cette donnée corporelle a lieu aussi dans son expressivité programmatique, dans les aspects d'adaptation de ces codes verbal, sonore et visuel, ses aspects épistémologiques aussi bien que la dichotomie entre diachronie et synchronie disparaissent. Nous ne pouvons pas oublier que cette écriture est engendrée par un programme, qui doit être préconçu comme écriture pour exister. Nous étions habitués, par la culture technologique antérieur, à usurper le côté pratique et performatif du code, mais si nous restons attentifs à ce concept nous nous rendons compte que programmer est créer une performance. Cette expansion inter-codes a permis, par l'intermédiaire de la nomenclature de programmation (stage, cast, member, behavior) de logiciels tels que Director MX de Adobe, l'option de programmer l'action et la simulation.

Nous commençons à modifier la façon d'acquérir les connaissances, le concept d'écrire, d'ouvrir ce genre de poésie. En plus d'être dans l'âge de l'expérimentation, nous sommes fruit de tout cela, modifiant le principal de cette histoire, à savoir : Qu'est-ce qu'être poète ? Je n'ose pas répondre ici, mais je me sens à l'aise pour dire que l'art et la poésie gagnent de plus en plus un même espace dans cette expansion de signes, en réduisant la distance, l'espace entre 
eux. Rien dans le milieu numérique ne se manifeste de façon isolée, il n’y a pas de réintroduction d'éléments chaogènes ou redondants, tout est fruit d'une écriture programmée, une écriture en expansion.

La poésie numérique, aussi incroyable qu'il puisse paraître, reprend la ritualisation du langage, car elle est un faire préprogrammé qui est prévu par son écriture et c'est ce crédit qui l'émancipe en tant que nouveauté. Toute pratique de cet exercice devient poétique, une poétique qui ne survit pas sans être précédée par une écriture. Dans cette circonstance, le poète numérique travaille avec les contradictions de ce programme numérique, concept développé - Looppoesia ${ }^{3}$-, en puisant dans cette écriture mathématique randomisée, mais figée, ce qu'elle peut nous offrir en tant que signe de lecture et d'interprétation de langages de programmation. Le poème peut donc être dépourvu de mots, car l'énonciation n'est pas dans le discours, le récit ne raconte pas d'histoire. Ce qui est poétique est l'expansion des signes : faire de la poésie numérique est construire des environnements - des ambiances - en mutation constante, une expérience qui n'a pas le souci de créer de formules.

Le mot aujourd'hui suit un processus qui ne peut être plus le seul signe qui représente l'objet des connaissances. Dans le monde cyber la mémoire n'appartient pas à l'espèce humaine : qui est le sujet qui parle, enfin, qui est le sujet? Les connaissances et leur acquisition découlent du savoir : elles ont créé une culture sémiotique transformant le concept $d^{\prime}$ intellection.

\footnotetext{
${ }^{3}$ Looppoesia: A Poética da Mesmice CD Rom, Wilton Azevedo, Mackpesquisa, São Paulo, 1994
} 
La transformation qu'a soufferte l'acquisition des connaissances scientifiques en d'autres domaines a rendu le dialogisme scientifique une prémisse pour de nouvelles découvertes. Ce que nous connaissons alors par langage appartient dorénavant à la biologie, la physique, la chimie..., dans le sens de comprendre son processus logique et de signes de la matière.

Par contradiction, et un positivisme persistant, la linguistique est demeurée éloignée de ces changements, sauf les études de Norbert Wiener", Max Bense et Claude Shannon, rien que pour nommer quelques-uns qui ont contribué, par leurs découvertes, à l'étude de la théorie de l'information et à celle des fréquences des mots, une étude qui a eu de l'importance pour l'éclosion de la Poésie Concrète, de la Poésie Visiva en Italie, et des Calligrammes, mais qui n'a envahi ni le monde de l'écrite ni celui de la littérature, comme nous pensions qu'il pourrait arriver.

Dans ce sens, la poésie numérique est la dernière instance pour servir de démarcation à la signification linguistique. La poésie numérique ne la limite pas, parce qu'elle fait partie d'un système qui se produit dans plusieurs directions, pareil à notre quotidien. Il n'y a rien de spécifique dans son discours, et voilà la difficulté de repérer son comportement linguistique.

La diversité de l'ambiance numérique, qui crée des conditions pour l'apparition de l'écriture en expansion, peut être 4 Le fondateur de la cybernétique, Norbert Wiener, avait déjà observé qui n'existe aucune opposition fondamental entre les problèmes qui se présentent aux spécialistes de la communication et ceux qui se posent aux linguistes. Pour les ingénieurs, il s'agit de transmettre un message à l'aide d'un code, c'est-à-dire, d'un numéro minimum de décisions binaires, autrement dit, $d^{\prime}$ un système de classification ou, enfin, d'un schéma qui représente les structures invariables. 
ambiguë pour ceux qui ne comprennent pas ce langage n'appartenant pas seulement aux signaux verbaux comme le dit Julia Kristeva :

On a soulevé plusieurs fois la question de savoir $s^{\prime}$ il existe un langage sans pensée et une pensée sans langage (Kristeva, 2007, page 16).

Il ne s'agit pas d'avoir une pensée qui ne concerne pas la linguistique, ce code-ci ou celui-là, mais plutôt de savoir comment demeurera l'acquisition du savoir poétique dans ces nouveaux systèmes de production de langage sémiotique.

Le savoir poétique est l'acte cognitif qui nous fait partenaires de ce processus. Nous commençons à articuler d'autres systèmes de signes concernant les échanges de ces signes, qui ne sont plus diachroniques dans le jeu sémiotique de la poésie numérique. Dans ma compréhension, cet échange a lieu dans un système plus triadique que dual, issu du matriciel du langage. La condition de la programmation qui est encore le principe qui engendre la signification, ne fixe pas la qualité de cette écriture.

\subsection{WLADEMIR DIAS PINO et LE POÉME PROCESSUS}

Comme le signale James O'Donnell (2000), même avec l'invention de l'imprimerie et avec tous les changements technologiques de la reproduction, nous n'avons pas réussi à modifier certaines choses essentielles de ce dispositif qu'est le livre:

A pesar de que hubo interrupciones profundas, la comunidad fundamental de productores $y$ usuarios de textos permaneció bastante fija: el clero y los aristócratas. Algunos ex monjes se convirtieron seguramente en profesores de universidad (Lutero es uno ejemplo claro), pero la continuidad de la 
comunidad de textos se intensifico al máximo. El códice conservo la forma externa del libro y las técnicas que explotaron su poder en los últimos años de una cultura exclusivamente manuscrita fueron perfeccionadas $y$ no sustituidas. (O'Donnell, 2000: 49-50)

Les expérimentations ont toujours été ouvertes au dialogisme entre la mesmice et l'étrangeté, et une fois que ce conflit disparâ̂t après le langage numérique demeurer comme supporte de la production poétique, il y a aujourd'hui un espace international contemporain, surtout après les poèmes concrets.

Le souci concernant la poésie et ses conséquences poétiques se présente toujours à l'égard de leurs structures et de combien la nouveauté dans la création de signes lui apporterait pour être acceptée comme avant-garde.

Wlademir Dias Pino (Dias Pino S/d) ouvre cette discussion en ayant mis son accent davantage sur son époque, quand il commence à développer l'idée du poème processus en tant que concept de déplacement, dans lequel il propose que le besoin d'un poème d'avoir des variations de sa propre solution impose au processus poétique d'admettre différentes structures de dispositif: le processus devient le poème et non plus la poésie :

Il $n^{\prime} y$ a pas de poésie-processus. Ce qui existe est le poèmeprocessus, car ce qui est un produit, $c^{\prime}$ est un poème. Celui qui enferme le processus est le poème. C'est le mouvement ou la participation créative qui amène la structure (matrice) à la condition de processus. Le processus du poète est individualiste, et ce qui intéresse collectivement est le processus du poème (Dias Pino, $\mathrm{S} / \mathrm{d}$ ). 
Pour moi, en faisant une analyse plus attentive dans ce qui concerne le concept d'hypermédia, tout ce que le poème processus porte en lui est lié aux variables informationnelles, pour que le poème atteigne un stade d'autophagie et qu'il soit capable de s'auto dépasser. Pour ne plus compter avec l'importance de l'ordre alphabétique, dans ce cas, le mot n'est plus nécessaire, ce qui le rend plus ouvert, et il ne se fixe plus spécifiquement en tant que langue :

Il ne s'agit pas, comme certains pourraient le penser, $d^{\prime}$ un combat rigide et gratuit contre le signe verbal, mais d'une exploration planifiée de possibilités comprises en d'autres signes (non verbaux). Il est intéressant de rappeler qui même les structures ne se traduisent pas : elles sont codifiées par les processus qui visent à la communication internationale. (Dias Pino, s/d)

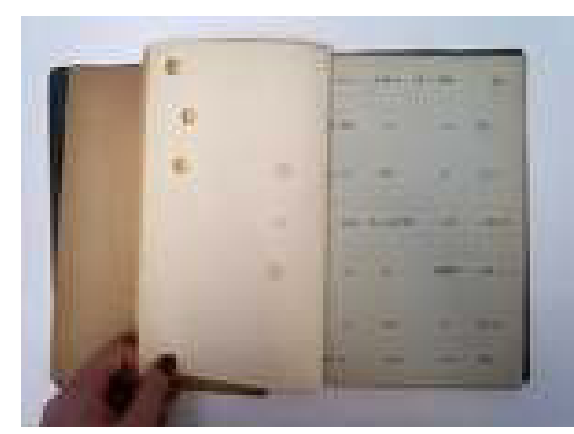

Livre-objet AVE de - A AVE de Wlademir Dias Pino - Cuiabá, Brasil, Igrejinha, 1956.

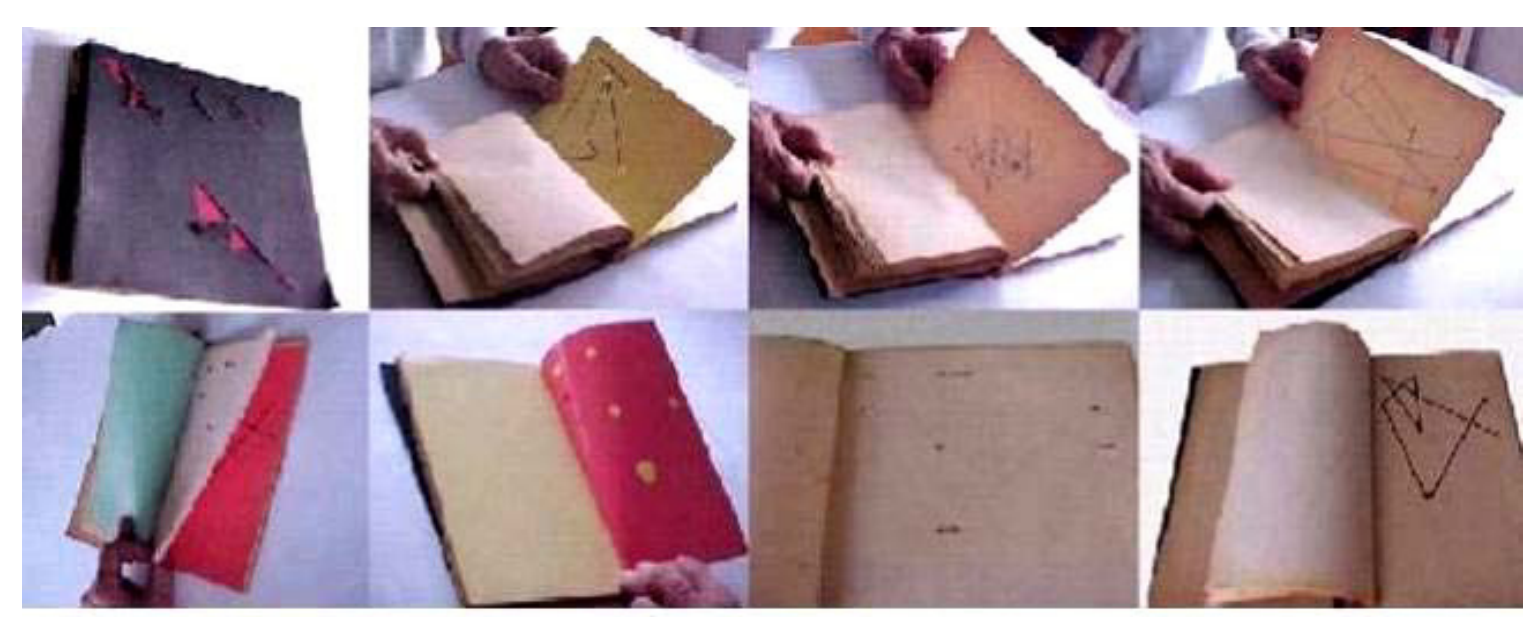

La Manipulation du Livre-objet A AVE de Wlademir Dias Pino 


\subsection{Poésie ou poème numérique}

Cette affirmation est d'extrême importance parce que Dias Pino développe ici l'idée que l'écriture n'appartient pas seulement au code verbal. Je sais qu'aujourd'hui c'est presque redondant de faire une telle affirmation, mais elle est issue de ce livre publié en $1972^{5}$ et qui avait déjà été écrit bien avant. C'est ici qui nous avons des indices de que sa poésie - ses idées - s'approche plutôt du concept d'hypermédia que de la poésie concrète, à tel point qu'il se laisse aller dans le risque d'une taxonomie qui nous amène à penser le poème numérique et sa programmation comme processus.

Dias Pino démontre comment peut opérer, du point de vue sémiotique, un indice qui présente le sens d'écriture:

Code : partie - stade d'un poème structure satellite: retardateur (de lecture mécanique du cerveau humain pour une information géométrisé : électronique)

Combinaison : une relativité.

Mot : Fixation verbal

Slogan : Phrase-verbal

Fragmentation : Utilisation de détails de lettres-explosion typographique.

\footnotetext{
5 J'attire attention que pour toute citation se rapportant à Wlademir Dias-Pino, son livre Processo: Linguagem Comunicação. Editora Vozes, Petrópolis, Rio de Janeiro, $\mathrm{n}^{\prime}$ a pas de référence d'année, ni numéro de page, ce que rend difficile préciser la source. Ce livre est cependant de 1972, d'après ce que l'auteur m'a dit dans une interview accordée à Rio de Janeiro en mars 2006 à l'Hôtel Othon Leme.
} 
Didactique : Pour l'apprentissage du poème.

Poèmes sans mot: Poèmes non typographiques (fin du modernisme) .

Nouvelles graphies : charades/tests.

Graphiques : géométrisation ; animation/circuit : exercices formés

d'image /collage; documents quotidiens.

À partir de ces concepts taxinomiques développés par Wlademir Dias Pino en ce qui concerne le poème processus, je propose ici une actualisation vers l'environnement numérique :

Code : Registre mémoriel,

Exercice poétique

Expansion de signes

Écriture Numérique : programmation /logiciel

Avatar : Une entité créée dans un milieu digital qui assume un corps de signes.

Relation de participation : Ambiance passive, réactive et interactive.

Opération : Articulation et non manipulation.

Manipulation : Physique, matérialité.

Articulation : virtuelles, signaux, syntaxe, écriture de programmation générative.

Processus Interactifs : 
Processus Interactif Cognitif : La Formation de signes mentaux interprétatifs ne modifie pas le processus d'articulation de l'émetteur, elle rend seulement les signes passibles d'interprétation, de/une volonté de significations établies par la syntaxe des signes.

Processus : Auto-acquisition

Processus Interactif $\mathbf{d}^{\prime}$ Intersection: Ensemble de signes où un code fait l'intersection de l'autre - il se configure par deux ou plusieurs codes qui se superposent - le changement d'articulation a lieu par permutation de cette intersection intersigne ${ }^{6}$.

Processus : Acquisition collective.

Processus Interactif $d^{\prime}$ Intervention : Processus de signes en expansion en intégrant espace/temps dans tous les codes. Cet item modifie, il interfère, il dilate, en empêchant le maintien d'une signification stable.

Processus : Acquisition Transitoire.

Cet indice nous permet de penser qu'un poème se fait d'une expérience, et je dirais encore : par l'expérimentation, en envisageant toute forme graphique, comme de nouvelles clés lexicales, en tant que processus qui s'auto-transforme, vu qu'il n'est pas rattaché seulement au mot ou signe indiciel,

Cf. Textes Complémentaires INTERPOESIA: DEFINITIONS, INDEFINItions, ANTECEDENTS ET CONSEQUENCES VIRTUELLES, de Philadelpho Menezes, qui on retrouve sur le CDRom Interpoesia 2000 - Mackenzie -São Paulo 
mais plutôt à l'iconique, c'est-à-dire, celui qui a une donnée imagée selon C.S.Peirce (1972).

Pour que l'on comprenne mieux, j'oserais proposer ici un petit schéma concernant tout ce qui a lieu dans les étapes de l'écriture numérique :

\section{Écriture Matricielle Numérique Lisible - (EMNL)}

Ces frontières matricielles, on peut les vérifier par l'histoire même du code adopté, qui s'est servi d'une technologie pour devenir une écriture. Par exemple, l'invention de l'écriture comme forme de mémorial de l'histoire, le magnétophone comme mémorial de l'oralité, l'appareil photo comme mémorial visuel, le cinéma comme mémorial du mouvement.

Ainsi les codes se sont servis et se servent encore de leur crédibilité non seulement comme mémorial, mais dans l'exercice de la crédibilité, et également dans la forme poétique.

Les frontières établies par l'écriture matricielle, même si elles ne créent pas une parataxe - chevauchement - vont permettre un progrès vers un autre modèle de signes intercodes. Le dialogisme existe ici, une fois que s'est établi un contact avec un autre code.

Cette forme d'écriture porte en soi toute la culture de registre mémoriel humain, par l'intermédiaire de ses signes conventionnels chiffrés : verbal/fixe ou mobile, imagé/2D ou 3D, et sonore/musical. Ces codes séparés par convention de 
leur propre histoire ont eu, chacun, pour leur visibilité et compréhension, leur technologie employée pour leur dissémination.

Les moyens d'information, tels que les journaux virtuels, travaillent avec ce concept, par la tradition même de l'environnement imprimé qui a conquiert sa crédibilité : le son, l'image et le texte sont disposés de façon bien claire et leur matrice est opérée de façon indicielle, autrement dit, de manière à ressembler au journal imprimé.

\section{Écriture Matricielle Numérique Intermédiaire (EMNI)}

Dans ce cas, les frontières établies par l'écriture matricielle ont aidé à promouvoir une parataxe dans l'écriture numérique - des signes qui opèrent par chevauchement. Cette progression n'est permise que pour ce deuxième item. Le contact se fait par intersection de deux codes.

Ici nous remarquons déjà une opération intercode d'un code qui pénètre un autre code de manière intersectée, où l'un prend de l'autre, les caractéristiques de signes qui ne font pas nécessairement partie de sa matrice. L'intermédiation à laquelle je fais référence, n'est pas quelque chose qui change la configuration, déforme la perception des trois codes en question, lesquels peuvent encore être détectés dans leurs caractéristiques. C'est juste l'intersection entre eux qui crée une espèce de poly-sémantisme, pourtant bien plus complexe, car cette intermédiation a lieu dans l'ambiance numérique d'un programme. 


\section{Écriture Numérique en Expansion (ENE)}

Cette dernière est, pour moi, celle qui caractérise la plupart des poésies numériques. Il n'y a plus de code prédominant ; l'ambiance est son-texte-image, ou comme l'a dit une fois Décio Pignatari une poésie «verbivocovisuelle ». Je sais qu'il faisait référence à une partie de la poésie concrète qui a toujours eu lieu dans l'espace bidimensionnel, mais qui convient ici, parce que ses matrices chiffrées disparaissent, survivent en forme de programmation admettant le processus en tant qu'écriture numérique en expansion, par la façon même dont le programme opère, sans distinction entre le son, l'image et le texte.

On remarque dans cette triade qu'il n’y a pas de signes prêts, définis par le symbolique qu'il répercute ou instaure. Les signes sont overlappings qui se refont en processus redondants de sémiose ou qui surgissent en tant que phénomènes phénoménologie peircienne - et ils présentent à chaque instant une circonstance toute neuve et inespérée. Les manifestations de langages et leurs productions poétiques dans le processus d'expansion dans cet environnement, qui n'est plus bidimensionnel, nous placent face à un signe qui n'est jamais prêt, à un interprétant final qui restaure sa qualité de signe - iconique -, à un signe qui est en expansion dans une direction non fermée, brisant ainsi la chaine de l'altérité, l'Autrui, ou comme le dit Tavares (1999), de l'image de l'étranger. 

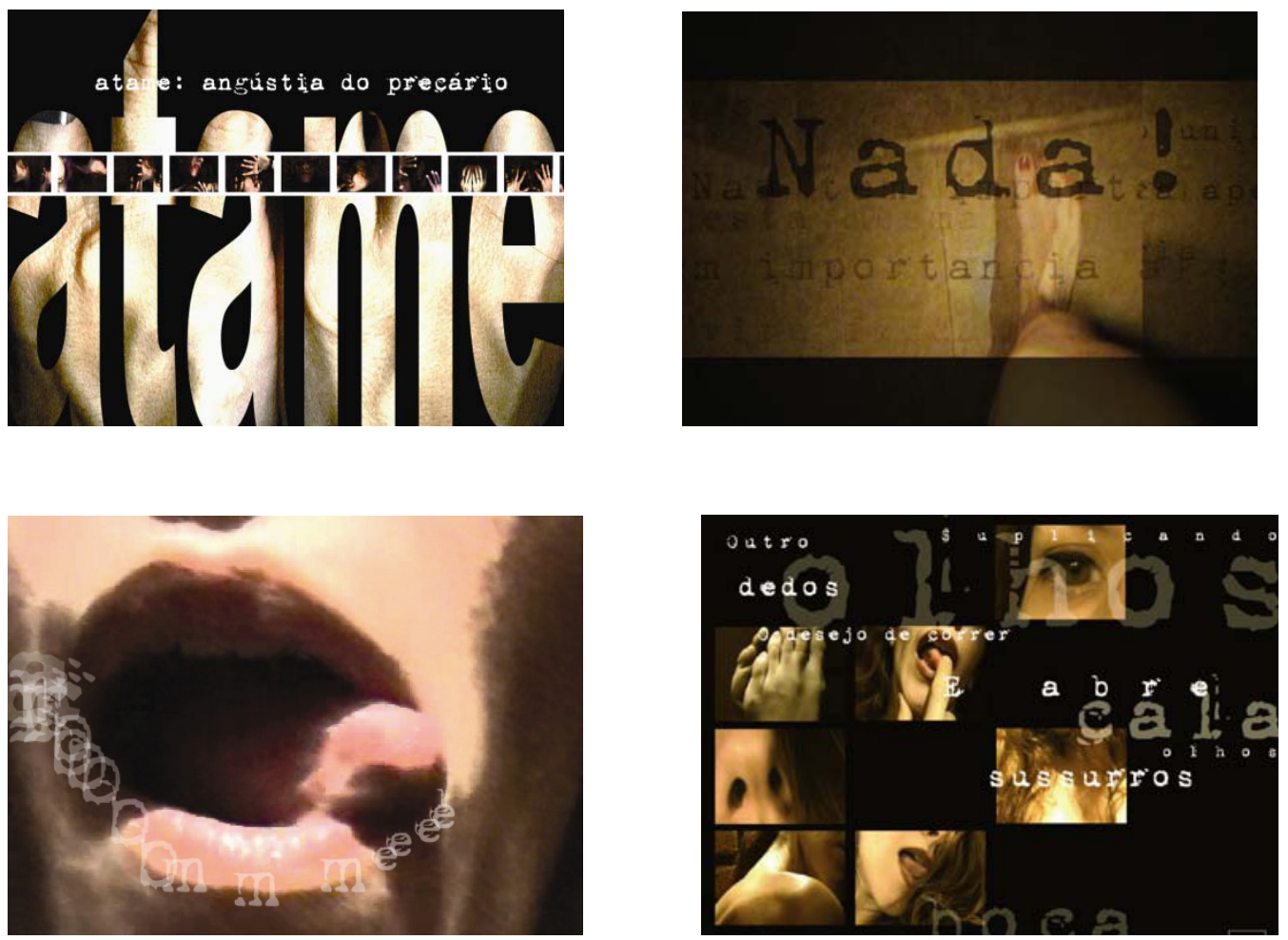

ATAME : L'angoisse du précaire - 2007/08 -Wilton Azevedo

L'imitation, la mimesis de l'ENE est un processus programmatique de pure simulation du faire et de représentation dans l'expérimenter. Je cherche ici à ne pas employer le verbe lire, puis qu'aujourd'hui ce verbe n'a plus la signification sui generis rattaché au code verbal. Ce que j'ai cependant appelé expérimentation au long de ce travail établit que l'acte de réception est aussi de nature en expansion.

Une carte routière, par exemple, pour toutes les fins pratiques, est $d$ 'une façon quintessent, une chose bidimensionnelle, un plan. Elle emploie deux dimensions pour transmettre des informations $d^{\prime}$ un genre précisément bidimensionnel. En vérité, bien sûr, les cartes routières sont tridimensionnelles, comme tout, mais leur épaisseur est si mince (et tellement sans relevance pour son but) qu'elle peut être oubliée. En effet une carte routière demeure bidimensionnelle, même lorsqu'elle est pliée. De la même manière, un fil est vraiment monodimensionnel, $c^{\prime}$ est une particule qui n'a aucune dimension (Greick, 1991, page 92). 
La poésie numérique ne se préoccupe pas d'un but, dans le sens du défi conclusif, mais elle a plutôt le souci d'être une ligne en expansion à la recherche de l'expérimentation.

Les locaux pour l'habitation étaient appelés mégaron polythyron, composés, en bref, de deux espaces qui communiquent entre eux par une série de portes qui, si elles veulent rester ouvertes, créeront un seul environnement (Santarcangeli, 1997, page 91).

La poésie numérique laisse plus évident à chaque étape, que la redondance de ses articulations de signes se fait présente en tant que poétique. Elle n'est pas pourtant la redondance engendrée par la culture POP, mais plutôt l'exhaustion, l'excès, en tant que processus d'étrangeté créant des extensions de ses signes sans plus avoir besoin de compter les mots.

Il y a toute une trajectoire de spéculations autour du comportement interdisciplinaire et des combats engagés par la survivance de la science et de la poétique. J'observe néanmoins que, dans ce siècle, il sera inévitable que nous pensions à de nouveaux outils d'analyse, parce que la poésie numérique en tant que produit hypermédia et de nature plurielle comporte d'autres sciences outre la sémiologie linguistique. Dans tout ce que nous comprenons par discours, nous pouvons encore dire que nous faisons de la poésie, et ce faire est un processus sémiotique. La recherche maintenant doit se tourner vers l'expérimentation poétique qui grave sa signification, raison par laquelle nous nous acculturons, c'est-à-dire, les codes et leurs dispositifs traditionnels $s^{\prime}$ acculturent. 
Toute invention de performance de l'avant-garde poétique, le temps et l'espace - chronotropo poétique - la poésie a été soumise pendant des milliers d'années dans l'espace bidimensionnel du papier, même si plus tard les nouveaux environnements électro-électroniques ayant surgi, leur spatialité a toujours été une sorte d'illusion de notre perception.

\section{BIBLIOGRAFIE}

DIAS PINO, Wlademir. Processo: linguagem e comunicação. Rio de Janeiro: Vozes.

GREICK, James. Caos: A criação de uma nova ciência, Tradução de Waltensir Dutra Rio de Janeiro: Campus, 1991.

KRISTEVA, Julia. História da Linguagem. Edições 70 Portugal, LisboaLAUAND, Luiz Jean (Org.). Cultura e educação na Idade Média. São Paulo: Martins Fontes, 1998.

O'DONNELL, James. Avatares de la palabras: del papiro al ciberespacio. Buenos Aires: Editorial Paidós, 2000.

PIERCE, Charles Sanders. Semiótica. Tradução José Teixeira Coelho Neto, Coleção Estudos. São Paulo: Perspectiva, 1977.

SANTARCANGELI, Paolo. El libro de los laberintos: historia de um mito y de um símbolo. Prólogo de Humberto Eco. Ediciones Siruela, 1997.

TAVARES, José, Fernando. Para uma Poética da Leitura. Literatura e Teoria. Universitátria Editora. Lisboa, 1999.

ZUMTHOR, Paul. Performance, recepção, leitura. 2a ed. Tradução Jerusa Pires Ferreira e Suely Fenerich. São Paulo: Cosac Naify, 2007. 